The authors reported no conflicts of interest.

The Journal policy requires editors and reviewers to disclose conflicts of interest and to decline handling or reviewing manuscripts for which they may have a conflict of interest. The editors and reviewers of this article have no conflicts of interest.

Regarding the first point, we reported ${ }^{4}$ the use of the preoperative ECMO support for those patients who had pericardial tamponade at presentation complicated with cardiac arrest. All these patients underwent ECMO by peripheral cannulation during cardiopulmonary resuscitation, and it was not possible to drain pericardial blood before restoring blood circulation by ECMO institution. Among these patients, brain death was a consistent cause of death, and it was not possible to verify potential weaning and the clinical efficacy of ECMO therapy. Moreover, multivariable analysis identified only cardiac arrest at presentation as an independent predictor of in-hospital mortality (odds ratio, 11.7, 95\%; confidence interval, 2.352-59.063; $P=.003$ ). Having said that, we agree with Uchida and colleagues regarding the utility of rapidly draining the pericardial blood to improve the cardiac output and to reduce the cerebral venous pressure. However, pericardial blood drainage is feasible in those patients with cardiac tamponade syndrome who still have myocardial contractile activity despite high doses of inotropic and vasoconstrictor drugs and instead not in those patients undergoing manual or automatic external cardiac massage in whom the absolute priority is to re-establish the systemic circulation very quickly.

Uchida and colleagues have correctly highlighted our statement ${ }^{4}$ regarding the absence of any benefit of perioperative ECMO on in-hospital survival in patients with cardiac arrest. However, it is worthy to note that $17.4 \%$ of patients supported by preoperative ECMO for cardiac arrest had a good outcome. This is the reason why we emphasized ${ }^{3}$ the use of ECMO to offer an immediate support and stabilization in those patients who presented with cardiac arrest.

Regarding the second point discussed by Uchida and colleagues, their classification of LVFWR in 3 groups is shareable. However, the difference between the oozing and sealed rupture is not always discernible during surgery, as is the case between blow-out and sealed rupture, the latter as a clear clot apposition on the site of rupture offering a temporary seal. Hence, in terms of surgical approach, we prefer to consider the classical 2 pathologic findings of LVFWR, preferring the sutureless technique ${ }^{3,4}$ in such cases of clear operative evidence of oozing rupture.

Unfortunately, cardiac arrest following LVFWR is a poor prognostic factor, and a prompt diagnosis together with a

rapid stabilization therapy and management are crucial to reduce the in-hospital complications and mortality in patients with LVFWR following acute myocardial infarction. ECMO implant may restore the organ perfusion and might change the otherwise-bad outcome in these very compromised and sick patients.

Francesco Formica, $M D^{a}$ Stefano D'Alessandro, MD, FECTS ${ }^{b}$

${ }^{a}$ Cardiac Surgery Unit Department of Medicine and Surgery Parma General Hospital University of Parma

Parma

${ }^{b}$ Cardiac Surgery Unit Cardiac-Thoracic-Vascular Department San Gerardo Hospital Monza, Italy

\title{
References
}

1. Uchida K, Yasuda S, Masuda M. Is preoperative ECMO effective for collapsed patients with left ventricular free wall rupture after myocardial infarction? $J$ Thorac Cardiovasc Surg. 2021;161:e34-5.

2. Okamura H, Kimura N, Mieno M, Matsumoto H, Yuri K, Yamaguchi A. Sutureless repair for postinfarction left ventricular free wall rupture. J Thorac Cardiovasc Surg. 2019;158:771-7.

3. Formica F, D'Alessandro S, Singh G. Left ventricular free wall rupture after myocardial infarction: still a challenging complication. J Thorac Cardiovasc Surg. 2019;158:e97-8.

4. Formica F, Mariani S, Singh G, D'Alessandro S, Messina LA, Jones N, et al. Postinfarction left ventricular free wall rupture: a 17-year single-centre experience. Eur J Cardiothorac Surg. 2018;53:150-6.

https://doi.org/10.1016/j.jtcvs.2020.08.063

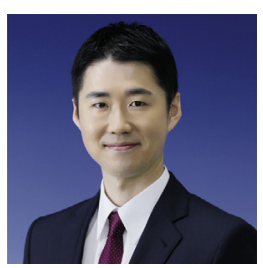

\section{REPLY FROM \\ AUTHOR: IS \\ EXTRACORPOREAL MEMBRANE OXYGENATION \\ USEFUL IN CARDIAC TAMPONADE?}

\section{Reply to the Editor:}

I appreciate the insightful letter by Uchida and colleagues ${ }^{1}$ regarding our group's previous articles about sutureless repair of left ventricular free wall rupture (LVFWR) and subsequent discussion with Formica and colleagues. ${ }^{2,3}$

Extracorporeal membrane oxygenation (ECMO) has been widely used to stabilize hemodynamics rapidly. As Uchida colleagues ${ }^{1}$ argued, however, some patients with LVFWR who have cardiac tamponade do not survive even with ECMO. In the study by Formica and colleagues, ${ }^{4} 6$ of the 8 nonsurvivors with preoperative ECMO died of brain death. Although some of the brain deaths might have already occurred before ECMO, there is a concern 
The author reported no conflicts of interest.

The Journal policy requires editors and reviewers to disclose conflicts of interest and to decline handling or reviewing manuscripts for which they may have a conflict of interest. The editors and reviewers of this article have no conflicts of interest.

that ECMO alone is not sufficiently effective to maintain cerebral blood perfusion under cardiac tamponade. The possible reason could be that tamponade causes high venous pressure, resulting in subsequent low systemic and cerebral perfusion pressures. Uchida and colleagues ${ }^{1}$ therefore suggested that drainage for pericardial hemorrhage rather than ECMO should be performed in patients with cardiac tamponade. Their argument is reasonable and noteworthy; however, the question remains as to how drainage should be performed in pericardial hemorrhage. Percutaneous pericardiocentesis can be promptly performed. A large blood clot around the heart, however, would usually make drainage of pericardial blood difficult. In such cases, surgical drainage through the subxiphoid can be an option; however, this needs expertise and might not be practical at institutions where cardiac surgeons are not readily available or during transport of the patient. Furthermore, increased intrapericardial pressure as a result of tamponade restrains the bleeding to some extent, and pericardial drainage can suddenly increase blood pressure and cause rebleeding as a result of LVFWR. This may reflect the wide use of ECMO without pericardial drainage in patients with cardiac tamponade.

Another possible solution under ECMO in cardiac tamponade might include placing the tip of the femoral venous cannula in the superior vena cava rather than the inferior vena cava. This might promote venous drainage through the superior vena cava and improve cerebral perfusion.

LVFWR has been traditionally classified into 2 types, blowout and oozing, although other morphologic types of rupture have been proposed. ${ }^{5}$ I agree that spontaneous sealing of the rupture site occurs in some patients during surgery. Our article classified sealed rupture as oozing rupture. ${ }^{1}$ It seems to me that distinguishing clearly between the oozing and sealed types of LVFWR is challenging. LVFWR usually occurs in elderly patients and those with comorbidities, and the myocardium is often covered with epicardial fat. When LVFWR occurs in the heart with excessive epicardial fat, oozing of blood may continue under the epicardial fat, even without the sign of bleeding outside the heart, in which case it becomes difficult to classify accurately. In such cases, the range of epicardial hematoma, rather than the distinction between the sealed and oozing types of hematoma, might be associated more with the extent of the impaired myocardium and operative results. In our article, a large epicardial hematoma was one of the characteristics of the cases in which postoperative rerupture occurred. ${ }^{2}$ In a retrospective study, these 2 types are difficult to distinguish clearly on the basis of operative reports.

\section{Homare Okamura, MD, PhD \\ Department of Cardiovascular Surgery \\ Saitama Medical Center \\ Jichi Medical University \\ Saitama, Japan \\ Department of Cardiovascular Surgery Nerima Hikarigaoka Hospital \\ Tokyo, Japan}

\section{References}

1. Uchida K, Yasuda S, Masuda M. Is preoperative ECMO effective for collapsed, patients with left ventricular free wall rupture after myocardial infarction? J Thorac Cardiovasc Surg. 2021;161:e34-5.

2. Okamura H, Kimura N, Mieno M, Matsumoto H, Yuri K, Yamaguchi A. Sutureless repair for postinfarction left ventricular free wall rupture. J Thorac Cardiovasc Surg. 2019;158:771-7.

3. Okamura H. Reply: Optimal management of left ventricular free wall rupture considering the probability of rerupture. J Thorac Cardiovasc Surg. 2019;158: e99-100.

4. Formica F, Mariani S, Singh G, D’Alessandro S, Messina LA, Jones N, et al. Postinfarction left ventricular free wall rupture: a 17-year single-centre experience. Eur J Cardiothorac Surg. 2018;53:150-6.

5. Purcaro A, Costantini C, Ciampani N, Mazzanti M, Silenzi C, Gili A, et al. Diagnostic criteria and management of subacute ventricular free wall rupture complicating acute myocardial infarction. Am J Cardiol. 1997;80:397-405.

https://doi.org/10.1016/j.jtcvs.2020.07.112 